Expressi on of I ongi tudi nal thi $\mathrm{rd}$ - or der transport coefficient in terns of a par ameters and its val i di ty

\begin{tabular}{|l|l|}
\hline 著者 & $\begin{array}{l}\text { KAWAGUCH Sat or u, TAKAHASH Kazuhi ro, SATOH } \\
\text { Kohki }\end{array}$ \\
\hline $\begin{array}{l}\text { j our nal or } \\
\text { publ i cat i on t i t l e }\end{array}$ & Pl asma Sources Sci ence and Technol ogy \\
\hline vol une & 27 \\
\hline number & 8 \\
\hline page range & 085006 \\
\hline year & 2018 08-07 \\
\hline URL & ht t p: //hdl . handl e. net /10258/00009681 \\
\hline
\end{tabular}




\title{
Expression of longitudinal third-order transport coefficient in terms of $\alpha$ parameters and its validity
}

\author{
S Kawaguchi ${ }^{1,3}$ (1), K Takahashi ${ }^{2}$ (i) and K Satoh ${ }^{2}$ \\ ${ }^{1}$ Division of Engineering, Graduate School of Engineering, Muroran Institute of Technology, Muroran, \\ Hokkaido, 050-8585, Japan \\ ${ }^{2}$ Division of Information and Electronic Engineering, Graduate School of Engineering, Muroran Institute \\ of Technology, Muroran, Hokkaido, 050-8585, Japan
}

E-mail: ksatoh@mmm.muroran-it.ac.jp

Received 30 March 2018, revised 8 June 2018

Accepted for publication 3 July 2018

Published DD MM 2018

\section{Abstract}

The relation between the longitudinal third-order transport coefficient $\omega_{3}$ contained in the continuity equation for electrons and $\alpha$ parameters defined by arrival-time spectra of an electron swarm is deduced. Values of $\omega_{3}$ and the $\alpha$ parameters in $\mathrm{CH}_{4}$ gas and $\mathrm{SF}_{6}$ gas are calculated by Monte Carlo simulation, and then the values of $\omega_{3}$ are compared with those of the longitudinal third-order transport coefficient $\omega_{3}^{\prime}$ which are calculated from the $\alpha$ parameters to examine the validity of the deduced relation. The values of $\omega_{3}^{\prime}$ are found to excellently agree with those of $\omega_{3}$ below $500 \mathrm{Td}$ in $\mathrm{CH}_{4}$ gas and from $150 \mathrm{Td}$ to $700 \mathrm{Td}$ in $\mathrm{SF}_{6}$ gas, where the values of the effective ionisation coefficient are nought or quite small. The results suggest that values of $\omega_{3}$ can be obtained experimentally from arrival-time spectra measured by a double-shutter drift tube.

Keywords: third-order transport coefficient, electron transport coefficient, $\alpha$ parameters, Monte Carlo simulation

\section{Introduction}

The third-order transport coefficient $\boldsymbol{Q}$, which is called skewness by several groups and generally represented as a third-order tensor, is one of the electron transport coefficients and appears in the continuity equation of the number density of electrons $n(\boldsymbol{r}, t)$ at position $\boldsymbol{r}$ and time $t$ [1]:

$$
\begin{aligned}
\frac{\partial n(\boldsymbol{r}, t)}{\partial t}= & \overline{R_{\mathrm{i}}} n(\boldsymbol{r}, t)-\boldsymbol{W} \cdot \frac{\partial n(\boldsymbol{r}, t)}{\partial \boldsymbol{r}}+\boldsymbol{D}:\left(\frac{\partial}{\partial \boldsymbol{r}} \otimes \frac{\partial}{\partial \boldsymbol{r}}\right) \\
& \times n(\boldsymbol{r}, t)-\boldsymbol{Q}:\left(\frac{\partial}{\partial \boldsymbol{r}} \otimes \frac{\partial}{\partial \boldsymbol{r}} \otimes \frac{\partial}{\partial \boldsymbol{r}}\right) n(\boldsymbol{r}, t)+\cdots
\end{aligned}
$$

where $\overline{R_{\mathrm{i}}}$ is the effective ionisation frequency, $\boldsymbol{W}$ is the bulk drift velocity, and $\boldsymbol{D}$ is the bulk diffusion coefficient. When an electric field $\boldsymbol{E}$ is applied in the $z$-direction, the tensor $\boldsymbol{Q}$ forms

\footnotetext{
3 Research Fellow of Japan Society for the Promotion of Science.
}

the following structure [1-3]:

$$
\begin{aligned}
Q_{x a b} & =\left(\begin{array}{ccc}
0 & 0 & Q_{x x z} \\
0 & 0 & 0 \\
Q_{x x z} & 0 & 0
\end{array}\right), Q_{y a b}=\left(\begin{array}{ccc}
0 & 0 & 0 \\
0 & 0 & Q_{x x z} \\
0 & Q_{x x z} & 0
\end{array}\right), \\
Q_{z a b} & =\left(\begin{array}{ccc}
Q_{z x x} & 0 & 0 \\
0 & Q_{z x x} & 0 \\
0 & 0 & Q_{z z z}
\end{array}\right),
\end{aligned}
$$

where $a, b, c \in\{x, y, z\}$. The tensor's components $Q_{a b c}$ are often grouped as the transverse third-order transport coefficient $D_{3 \mathrm{~T}}=\left(Q_{z x x}+Q_{x x z}+Q_{x z x}\right) / 3$ and the longitudinal third-order transport coefficient $D_{3 \mathrm{~L}}=Q_{z z z}$. Equation (1) is often employed for the discharge plasma modeling, but the terms containing third-order and higher-order transport coefficients are generally ignored since little information on those transport coefficients is reported; however, it was pointed out that the consideration of the term containing the third-order transport coefficient may be important in modelling of discharge plasmas under the influence of high electric field [1]. 
Furthermore, since the right-hand side of equation (1) is an infinite series, considering the terms including third-order and higher-order transport coefficients is profitable to describe electron behaviour in plasmas accurately. Therefore, examination of the third-order transport coefficient has been carried out in recent years [1-5].

Penetrante and Bardsley [4] calculated values of $N^{2} D_{3 \mathrm{~L}}$ in $\mathrm{He}, \mathrm{Ne}$, and Ar below $E / N=4 \mathrm{Td}$ using electron collision cross sections by solving the Boltzmann equation and Monte Carlo simulation. Here, $N$ and $E / N$ denote the number density of gas molecules and the reduced electric field, respectively, and $1 \mathrm{Td}$ is equal to $10^{-17} \mathrm{Vcm}^{2}$. Petrović et al [1] calculated the components of the third-order transport coefficient tensor in $\mathrm{CH}_{4}$ gas from $0.1 \mathrm{Td}$ to $1000 \mathrm{Td}$ by solving the Boltzmann equation and Monte Carlo simulation. They reported that the values of $N^{2} D_{3 \mathrm{~T}}$ become negative in the range of $E / N$ where the negative differential conductivity appears whilst the values of $N^{2} D_{3 \mathrm{~L}}$ remain positive. In figures 3-6 shown in their paper, variations of the tensor components with respect to $E / N$ are found to not be simple and differ from those of the flux electron drift velocity and longitudinal diffusion coefficient. The electron transport coefficients are determined by electron collision cross sections, which are fundamental data to describe electron behaviour in gases; therefore, the validity of electron collision cross sections is often examined by comparing the transport coefficients calculated from the cross sections with measured data, and their report indicates that comparison between the measured and calculated third-order transport coefficient enables further examination of the validity of the electron collision cross sections. To examine the third-order transport coefficient in various gases and to verify their calculations, measurement of the third-order transport coefficient is indispensable; however, a method of measuring the third-order transport coefficient has not yet been established.

Kondo and Tagashira [6] introduced new electron transport coefficients called $\alpha$ parameters, which are defined by arrival-time spectra of an electron swarm, and reported that the $\alpha$ parameters are related to $D_{3 \mathrm{~L}}$. Arrival-time spectra of an electron swarm can be measured by a double-shutter drift tube, and the procedure for obtaining the $\alpha$ parameters from the measured arrival time spectra is well established [7-15]; therefore, there is a possibility that values of $D_{3 \mathrm{~L}}$ are obtained experimentally by the $\alpha$ parameters.

The aim of this work is to propose the new method for deducing the longitudinal third-order transport coefficient by using $\alpha$ parameters. The relation between the longitudinal third-order transport coefficient $D_{3 \mathrm{~L}}$ and the $\alpha$ parameters is deduced by using the theory of arrival time spectra established by Kondo and Tagashira. To examine the validity of the deduced relation, values of $D_{3 \mathrm{~L}}$ and the $\alpha$ parameters in $\mathrm{CH}_{4}$ gas and $\mathrm{SF}_{6}$ gas are calculated by Monte Carlo simulation, and the values of $D_{3 \mathrm{~L}}$ are compared with those of the longitudinal third-order transport coefficient which is defined by the $\alpha$ parameters.

\section{Definitions of $\omega$ and $\alpha$ parameters and their relations}

By integrating equation (1) with respect to $x$ and $y$, and defining that $n(z, t)=\int_{-\infty}^{\infty} n(\boldsymbol{r}, t) \mathrm{d} x \mathrm{~d} y$, the following equation is obtained.

$$
\begin{aligned}
\frac{\partial n(z, t)}{\partial t}= & \omega_{0} n(z, t)-\omega_{1} \frac{\partial n(z, t)}{\partial z}+\omega_{2} \frac{\partial^{2} n(z, t)}{\partial z^{2}} \\
& -\omega_{3} \frac{\partial^{3} n(z, t)}{\partial z^{3}}+\cdots
\end{aligned}
$$

Here, $\omega_{0}, \omega_{1}, \omega_{2}$ and $\omega_{3}$ respectively represent the effective ionisation frequency, bulk drift velocity, longitudinal diffusion coefficient and longitudinal third-order transport coefficient. Those transport coefficients are called $\omega$ parameters, and the definitions of the $\omega$ parameters are expressed as follows [16]:

$$
\begin{gathered}
\omega_{0}=\frac{\mathrm{d}}{\mathrm{d} t} \ln \int_{-\infty}^{\infty} n(z, t) \mathrm{d} z, \\
\omega_{1}=\frac{\mathrm{d}}{\mathrm{d} t} \bar{z}(t), \\
\omega_{2}=\frac{1}{2 !} \frac{\mathrm{d}}{\mathrm{d} t} \frac{\int_{-\infty}^{\infty}(z-\bar{z}(t))^{2} n(z, t) \mathrm{d} z}{\int_{-\infty}^{\infty} n(z, t) \mathrm{d} z}, \\
\omega_{3}=\frac{1}{3 !} \frac{\mathrm{d}}{\mathrm{d} t} \frac{\int_{-\infty}^{\infty}(z-\bar{z}(t))^{3} n(z, t) \mathrm{d} z}{\int_{-\infty}^{\infty} n(z, t) \mathrm{d} z}
\end{gathered}
$$

where $\bar{z}(t)$ is defined as

$$
\bar{z}(t)=\frac{\mathrm{d}}{\mathrm{d} t} \frac{\int_{-\infty}^{\infty} z n(z, t) \mathrm{d} z}{\int_{-\infty}^{\infty} n(z, t) \mathrm{d} z} .
$$

Kondo and Tagashira [6] introduced the following continuity equation:

$$
\begin{aligned}
\frac{\partial n(z, t)}{\partial z}= & \alpha_{0} n(z, t)-\alpha_{1} \frac{\partial n(z, t)}{\partial t}+\alpha_{2} \frac{\partial^{2} n(z, t)}{\partial t^{2}} \\
& -\alpha_{3} \frac{\partial^{3} n(z, t)}{\partial t^{3}}+\cdots,
\end{aligned}
$$

which is equivalent to equation (3). Here, $\alpha_{0}, \alpha_{1}, \alpha_{2}$ and $\alpha_{3}$ are called $\alpha$ parameters and defined as follows:

$$
\begin{gathered}
\alpha_{0}=\frac{\mathrm{d}}{\mathrm{d} z} \ln \int_{0}^{\infty} n(z, t) \mathrm{d} t, \\
\alpha_{1}=\frac{\mathrm{d}}{\mathrm{d} z} \bar{t}(z), \\
\alpha_{2}=\frac{1}{2 !} \frac{\mathrm{d}}{\mathrm{d} z} \frac{\int_{0}^{\infty}(t-\bar{t}(z))^{2} n(z, t) \mathrm{d} t}{\int_{0}^{\infty} n(z, t) \mathrm{d} t},
\end{gathered}
$$




$$
\alpha_{3}=\frac{1}{3 !} \frac{\mathrm{d}}{\mathrm{d} z} \frac{\int_{0}^{\infty}(t-\bar{t}(z))^{3} n(z, t) \mathrm{d} t}{\int_{0}^{\infty} n(z, t) \mathrm{d} t},
$$

where $\bar{t}(z)$ is defined as

$$
\bar{t}(z)=\frac{\int_{0}^{\infty} \operatorname{tn}(z, t) \mathrm{d} t}{\int_{0}^{\infty} n(z, t) \mathrm{d} t} .
$$

Here, $\alpha_{0}$ is equal to the effective ionisation coefficient measured by the steady-state Townsend experiment.

The relation between the $\omega$ and $\alpha$ parameters is expressed by equations (15)-(17) in terms of the infinite series $\Omega_{k}$ $(k=1,2,3)$ defined by equation (18) [6].

$$
\begin{gathered}
\alpha_{1}=\frac{1}{\Omega_{1}} \\
\alpha_{2}=\frac{\Omega_{2}}{\left(\Omega_{1}\right)^{3}} \\
\alpha_{3}=\frac{2\left(\Omega_{2}\right)^{2}}{\left(\Omega_{1}\right)^{5}}-\frac{\Omega_{3}}{\left(\Omega_{1}\right)^{4}} \\
\Omega_{k}=\sum_{i=k}^{\infty}(-1)^{i-k}\left(\begin{array}{l}
i \\
k
\end{array}\right)\left(\alpha_{0}\right)^{i-k} \omega_{i} .
\end{gathered}
$$

Equation (19) is deduced from equations (15)-(17).

$$
\Omega_{3}=\frac{2\left(\alpha_{2}\right)^{2}}{\left(\alpha_{1}\right)^{5}}-\frac{\alpha_{3}}{\left(\alpha_{1}\right)^{4}} .
$$

From equation (18), $\Omega_{3}$ is described as

$$
\Omega_{3}=\omega_{3}-4 \alpha_{0} \omega_{4}+10\left(\alpha_{0}\right)^{2} \omega_{5}-\cdots
$$

By substituting equation (19) for equation (20), $\omega_{3}$ is expressed as

$$
\omega_{3}=\frac{2\left(\alpha_{2}\right)^{2}}{\left(\alpha_{1}\right)^{5}}-\frac{\alpha_{3}}{\left(\alpha_{1}\right)^{4}}+4 \alpha_{0} \omega_{4}-10\left(\alpha_{0}\right)^{2} \omega_{5}+\cdots
$$

In general, the values of $\omega_{k}(k>3)$ are regarded as negligibly small in comparison with those of $\omega_{3}$; therefore, values of $\omega_{3}^{\prime}$ defined by equation (22) are considered to be approximately equal to those of $\omega_{3}$ when the values of $\alpha_{0}$ are small. When the values of $\alpha_{0}$ are nought, the values of $\omega_{3}^{\prime}$ are exactly equal to those of $\omega_{3}$.

$$
\omega_{3}=\frac{2\left(\alpha_{2}\right)^{2}}{\left(\alpha_{1}\right)^{5}}-\frac{\alpha_{3}}{\left(\alpha_{1}\right)^{4}} .
$$

\section{Simulation method and condition}

Electron transport in $\mathrm{CH}_{4}$ gas and $\mathrm{SF}_{6}$ gas under the influence of a uniform DC electric field $\boldsymbol{E}=(0,0, E)$ is simulated by the Monte Carlo method. Electron collisions with $\mathrm{CH}_{4}$ and $\mathrm{SF}_{6}$ molecules are only considered, and the number density of gas molecules set to $3.535 \times 10^{16} \mathrm{~cm}^{3}\left(0^{\circ} \mathrm{C}\right.$ and 1 Torr $)$, assuming the typical condition of the swarm experiments. Thermal motion of gas molecules, which may influence electron transport in gas under the influence of low reduced electric field, is considered in the calculation of electron velocity right after the collision. The flight time of an electron between successive collisions is calculated by the null-collision method [17]. The sets of electron collision cross sections for $\mathrm{CH}_{4}$ gas and $\mathrm{SF}_{6}$ gas respectively proposed by Šašić et al [18] and Itoh et al [19] are used. Arrival-time spectra of an isolated electron swarm are sampled, and the $\alpha$ parameters are calculated in accordance with equations (10)(13). The longitudinal second-order $M_{\mathrm{L}}(t)$, third-order $M_{3 \mathrm{~L}}(t)$ and forth-order $M_{4 \mathrm{~L}}(t)$ moments of electrons are respectively sampled in accordance with equations (23)-(25).

$$
\begin{aligned}
& M_{\mathrm{L}}(t)=\frac{1}{N_{\mathrm{e}}(t)} \sum_{i=1}^{N_{\mathrm{e}}(t)}\left(z_{i}(t)-\bar{z}(t)\right)^{2} \\
& M_{3 \mathrm{~L}}(t)=\frac{1}{N_{\mathrm{e}}(t)} \sum_{i=1}^{N_{\mathrm{e}}(t)}\left(z_{i}(t)-\bar{z}(t)\right)^{3} \\
& M_{4 \mathrm{~L}}(t)=\frac{1}{N_{\mathrm{e}}(t)} \sum_{i=1}^{N_{\mathrm{e}}(t)}\left(z_{i}(t)-\bar{z}(t)\right)^{4} .
\end{aligned}
$$

Here, $N_{\mathrm{e}}(t)$ is the number of electrons, $z_{i}(t)$ is the position of the $i$ th electron, and $\bar{z}(t)$ is the centre of mass of electrons sampled as

$$
\bar{z}(t)=\frac{1}{N_{\mathrm{e}}(t)} \sum_{i=1}^{N_{\mathrm{e}}(t)} z_{i}(t)
$$

Values of $\omega_{3}$ are calculated from equation (27). Furthermore, values of the fourth-order transport coefficient $\omega_{4}$ is calculated from equation (28) [20] in order to examine the contribution of the term $4 \alpha_{0} \omega_{4}$ in equation (21) on the values of $\omega_{3}$. The method of the simulation is described in detail in our previous papers [21].

$$
\begin{gathered}
\omega_{3}=\frac{1}{3 !} \frac{\mathrm{d}}{\mathrm{d} t} M_{3 \mathrm{~L}}(t) \\
\omega_{4}=\frac{1}{4 !} \frac{\mathrm{d}}{\mathrm{d} t}\left[M_{4 \mathrm{~L}}(t)-3\left(M_{\mathrm{L}}(t)\right)^{2}\right] .
\end{gathered}
$$

To deduce accurate values of $\alpha$ and $\omega$ parameters, Monte Carlo simulation is conducted five times at the same $E / N$ value using different random seeds, and values of those parameters are deduced from regression analysis of the sampled values. Uncertainty of those parameters is evaluated by taking the root mean square of residuals, and then weighted averages of those parameters are calculated. The initial number of traced electrons is set to $10^{5}-10^{7}$ in $\mathrm{SF}_{6}$ gas above $300 \mathrm{Td}$ and in $\mathrm{CH}_{4}$ gas. Because of the strong depletion of traced electrons by electron attachment collisions, the initial number of traced electrons is set to $10^{8}-10^{9}$ in $\mathrm{SF}_{6}$ gas at $300 \mathrm{Td}$ or less. The enormous initial electrons lead to high density of charged species, which disturbs the applied electric field. However, the electric field created by the charged species is not considered in our simulation; that is, the traced electrons are treated as superparticles, and downscaling of the particles is implemented implicitly. 


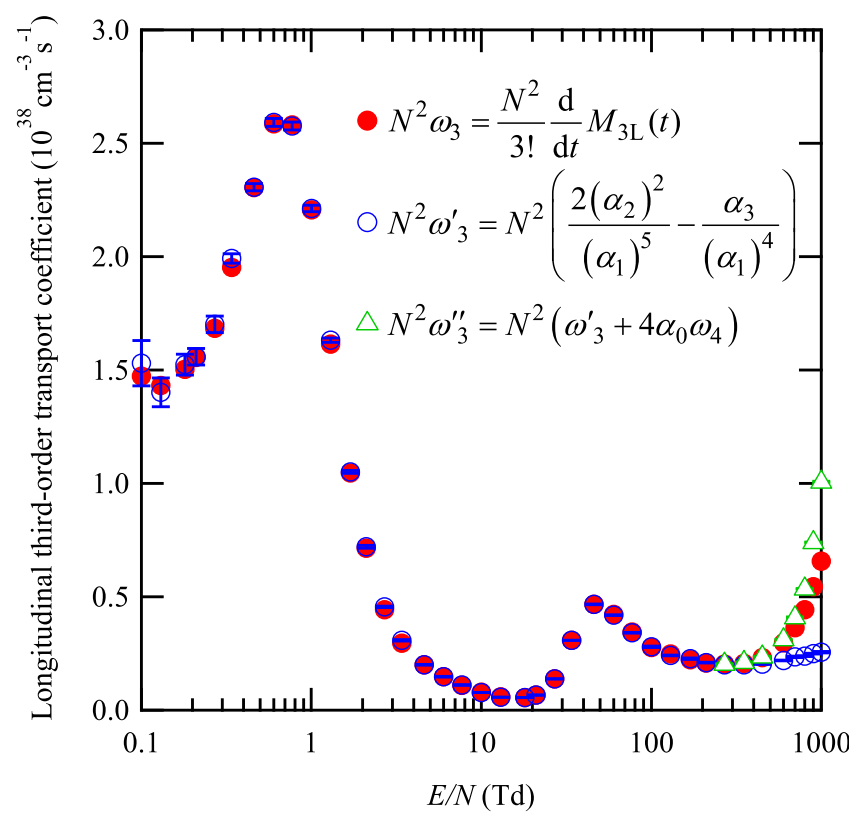

Figure 1. Longitudinal third-order transport coefficient in $\mathrm{CH}_{4}$ gas as a function of $E / N$.

\section{Results and discussion}

Figures 1 and 2 show the values of the longitudinal thirdorder transport coefficients $N^{2} \omega_{3}, N^{2} \omega_{3}^{\prime}$ and $N^{2} \omega^{\prime \prime}{ }_{3}$ and the effective ionisation coefficient $\alpha_{0} / N$ in $\mathrm{CH}_{4}$ gas as functions of $E / N$. Here, $\omega^{\prime \prime}{ }_{3}$ denotes $\omega_{3}^{\prime}+4 \alpha_{0} \omega_{4}$, which is a part of equation (21). The values of $N^{2} \omega_{3}^{\prime}$ are found to agree excellently with those of $N^{2} \omega_{3}$ below $500 \mathrm{Td}$. However, the values of $N^{2} \omega_{3}^{\prime}$ are lower than those of $N^{2} \omega_{3}$ above $500 \mathrm{Td}$, where the values of $\alpha_{0} / N$ increase exponentially with $E / N$. Since the values of $\omega_{4}$ and $\alpha_{0}$ are positive above $270 \mathrm{Td}$, the term $4 \alpha_{0} \omega_{4}$ in equation (21) contributes to increase the values of $\omega_{3}$. Therefore, the values of $N^{2} \omega^{\prime \prime}{ }_{3}$ are higher than those of $N^{2} \omega_{3}^{\prime}$ and found to agree with those of $N^{2} \omega_{3}$ up to $700 \mathrm{Td}$. This result suggests that the terms containing $\alpha_{0}$ in equation (21) cannot be ignored to calculate the values of $\omega_{3}$ accurately above $500 \mathrm{Td}$ in $\mathrm{CH}_{4}$ gas.

Figures 3 and 4 show the values of the longitudinal thirdorder transport coefficients $N^{2} \omega_{3}$ and $N^{2} \omega_{3}^{\prime}$ and the effective ionisation coefficient $\alpha_{0} / N$ in $\mathrm{SF}_{6}$ gas as functions of $E / N$. Since the number of electrons sampled in the simulation drastically decreases due to electron attachment collisions with $\mathrm{SF}_{6}$ molecules, uncertainty of the values of $N^{2} \omega_{3}^{\prime}$ is pronounced below $300 \mathrm{Td}$. This uncertainty is mainly derived from that of the values of $\alpha_{3}$ due to the statistical fluctuation of sampled values of $\bar{T}_{3}$. The values of $N^{2} \omega_{3}^{\prime}$ agree with those of $N^{2} \omega_{3}$ from $150 \mathrm{Td}$ to $700 \mathrm{Td}$; however, discrepancy between the values of $N^{2} \omega_{3}$ and $N^{2} \omega_{3}^{\prime}$ is found below $150 \mathrm{Td}$ and above $700 \mathrm{Td}$, indicating that the contribution of the terms ignored in $N^{2} \omega_{3}^{\prime}$ such as $4 \alpha_{0} \omega_{4}$ may be significant. Whilst the values of $\omega_{4}$ in $\mathrm{SF}_{6}$ gas could not be calculated due to the statistical fluctuation of the sampled values of $M_{4 \mathrm{~L}}(t)-3\left(M_{\mathrm{L}}(t)\right)^{2}$, those sampled values seem to decrease with $t$ below $200 \mathrm{Td}$ and above $300 \mathrm{Td}$, indicating that the values of $\omega_{4}$ are negative. Therefore, when the values of $\alpha_{0}$ are

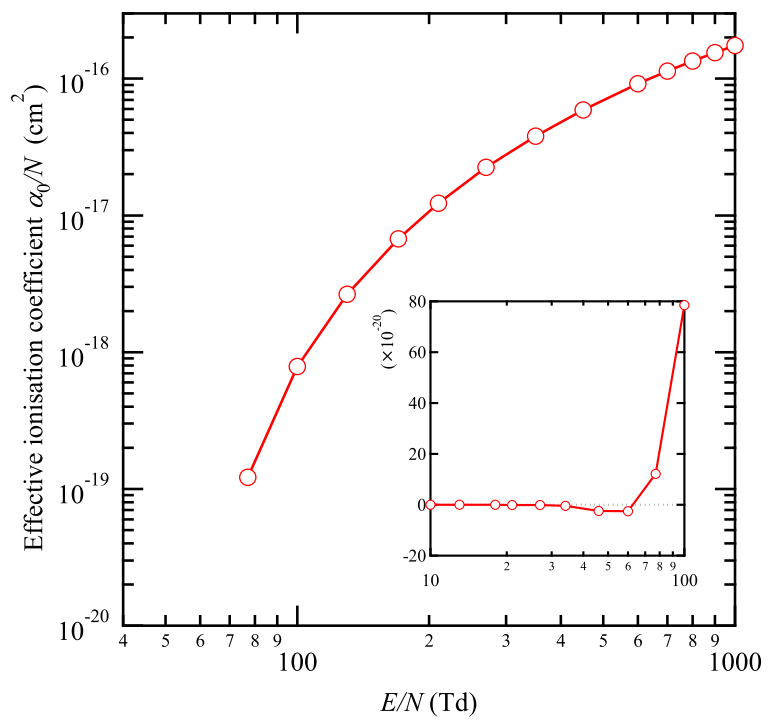

Figure 2. Effective ionisation coefficient in $\mathrm{CH}_{4}$ gas as a function of $E / N$.

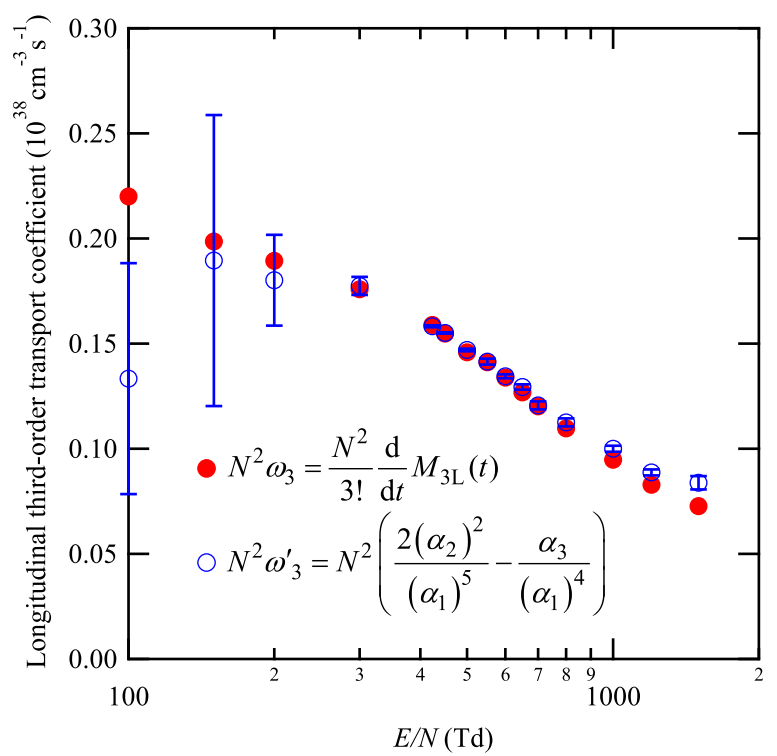

Figure 3. Longitudinal third-order transport coefficient in $\mathrm{SF}_{6}$ gas as a function of $E / N$.

positive, the term $4 \alpha_{0} \omega_{4}$ contributes to decrease the values of $\omega_{3}$, and as a result, the values of $N^{2} \omega_{3}^{\prime}$ are higher than those of $N^{2} \omega_{3}$ above $700 \mathrm{Td}$. On the other hand, the term $4 \alpha_{0} \omega_{4}$ contributes to increase the values of $\omega_{3}$ below $200 \mathrm{Td}$; therefore, the values of $N^{2} \omega_{3}^{\prime}$ are lower than those of $N^{2} \omega_{3}$ below $150 \mathrm{Td}$.

Calculated values of the longitudinal third-order transport coefficient in $\mathrm{CH}_{4}$ gas and $\mathrm{SF}_{6}$ gas are presented and discussed above. To understand and describe electron behaviour in gases accurately, further study regarding the nature of the longitudinal third-order transport coefficient and other components of the skewness tensor is required. Examination of the relation between the third-order transport coefficients and electron collision cross sections, for example, would be profitable to clarify the nature of the coefficients and may 


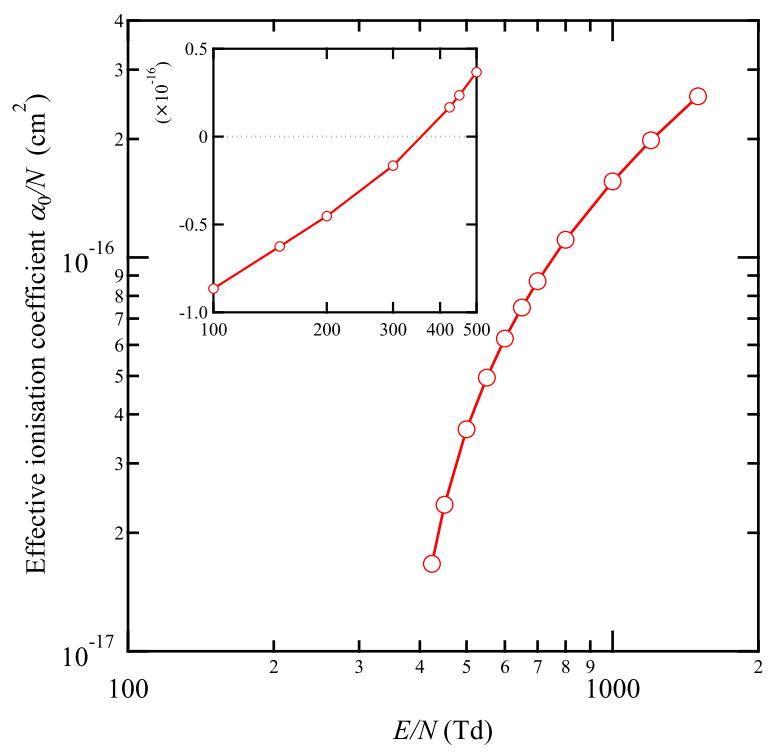

Figure 4. Effective ionisation coefficient in $\mathrm{SF}_{6}$ gas as a function of E/N.

provide a guide to determine the shape and magnitude of electron collision cross sections.

\section{Conclusions}

The relation between the longitudinal third-order transport coefficient $\omega_{3}$ and the $\alpha$ parameters is deduced based on the theory of arrival-time spectra of an electron swarm developed by Kondo and Tagashira. Furthermore, the values of the longitudinal third-order transport coefficient $\omega_{3}$ and $\alpha$ parameters in $\mathrm{CH}_{4}$ gas and $\mathrm{SF}_{6}$ gas are calculated by Monte Carlo simulation, and the values of $\omega_{3}$ are compared with those of the longitudinal third-order transport coefficient $\omega_{3}^{\prime}$ calculated from the $\alpha$ parameters. It is found that the values of $N^{2} \omega_{3}^{\prime}$ agree excellently with those of $N^{2} \omega_{3}$ up to $500 \mathrm{Td}$ in $\mathrm{CH}_{4}$ gas and from $150 \mathrm{Td}$ to $700 \mathrm{Td}$ in $\mathrm{SF}_{6}$ gas. This indicates that it is possible to obtain values of the longitudinal third-order transport coefficient experimentally by measuring arrival-time spectra of an electron swarm using a double-shutter drift tube in the range of $E / N$ where the values of the effective ionisation coefficient are nought or quite small.

\section{Acknowledgments}

This work was partly supported by JSPS KAKENHI Grant Number JP17J11124.

\section{ORCID iDs}

S Kawaguchi (10 https://orcid.org/0000-0003-3115-8719

K Takahashi (i) https://orcid.org/0000-0002-9358-0117

\section{References}

[1] Petrović Z L, Simonović I, Marjanović S, Bošnjaković D, Marić D, Malović G and Dujko S 2017 Plasma Phys. Control. Fusion 59014026

[2] Koutselos A D 2001 Chem. Phys. 270165

[3] Vrhovac S B, Petrović Z L, Viehland L A and Santhanam T S 1999 J. Chem. Phys. 1102423

[4] Penetrante B M and Bardsley J N 1990 Nonequilibrium Effects in Ion and Electron Transport ed J W Gallagher et al (New York: Plenum) pp 49-66

[5] Stokes P W, Simonović I, Philippa B, Cocks D, Dujko S and White R D 2018 Sci. Rep. 82226

[6] Kondo K and Tagashira H 1990 J. Phys. D: Appl. Phys. 231175

[7] Yoshida K, Ohshima T, Ohuchi H, Kishimoto Y and Tagashira H 1996 J. Phys. D: Appl. Phys. 292447

[8] Yoshida K, Ohshima T, Ohmori Y, Ohuchi $\mathrm{H}$ and Tagashira $\mathrm{H}$ 1996 J. Phys. D: Appl. Phys. 291209

[9] Yoshida K, Mori S, Kishimoto Y, Ohuchi H, Hasegawa H, Shimozuma M and Tagashira H 2005 J. Phys. D: Appl. Phys. 381918

[10] Yoshida K, Sato R, Yokota T, Kishimoto Y and Date H 2011 Japan. J. Appl. Phys. 50120210

[11] Hasegawa H, Date H, Shimozuma M, Yoshida K and Tagashira H 1996 J. Phys. D: Appl. Phys. 292664

[12] Hasegawa H, Date H, Ohmori Y, Ventzek P L G, Shimozuma M and Tagashira H 1998 J. Phys. D: Appl. Phys. 31737

[13] Hasegawa H, Date H and Shimozuma M 2007 J. Phys. D: Appl. Phys. 402495

[14] Hasegawa H, Date H, Shimozuma M and Itoh H 2009 Appl. Phys. Lett. 95101504

[15] Hasegawa H and Date H 2015 J. Appl. Phys. 117133302

[16] Kumar K, Skullerud H R and Robson R E 1980 Aust. J. Phys. 33343

[17] Skullerud H R 1968 J. Phys. D: Appl. Phys. 11567

[18] Šašić O, Malović G, Strinić A, Nikitović Ž and Petrović Z L 2004 New. J. Phys. 674

[19] Itoh H, Matsumura T, Satoh K, Date H, Nakao Y and Tagashira H 1993 J. Phys. D: Appl. Phys. 261975

[20] Makabe T and Lj P Z 2016 Plasma Electronics: Applications in Microelectronic Device Fabrication (Boca Raton, FL: CRC Press) p 153

[21] Kawaguchi S, Takahashi K, Satoh K and Itoh H 2016 Japan. J. Appl. Phys. 55 07LD03 